\title{
MAGNETO-OPTIC AND SCANNING PROBE MICROSCOPY STUDIES OF DIRECTLY PATTERNED MAGNETIC FILMS
}

\author{
N.I. Polushkin and K.V. Rao* \\ Institute for Physics of Microstructures of RAS, 603950 GSP-105 Nizhni Novgorod, Russia (e-mail: nip@ipm.sci-nnov.ru) \\ *Royal Institute of Technology, SE-10044 Stockholm, Sweden (e-mail: rao@kth.se)
}

\begin{abstract}
Using the magneto-optic Kerr effect and atomic force microscopy (AFM) we investigate nanoscale ferromagnetic entities directly patterned in thin superparamagnetic films of $\mathrm{Fe}-\mathrm{Cr}$ by means of interfering laser beams. We demonstrate, for the first time, that a contact mode of AFM with a non-magnetic tip can be suitably modified to visualize in great detail the response to external fields of even a single nano-ferromagnetic entity in a patterned array. This new approach for magnetic imaging, which we consider to be a magnetostrictive response (MSR) technique, having a lateral resolution typical of AFM, is used to show the strong dependence of the magnetic response of these nanoscale entities to prior magnetization direction. The observed MSR effects provide further insight into the magnetic microstructural aspects of the laser patterned entities and explains qualitatively the information obtained by means of Kerr magnetometry and magnetic force microscopy.
\end{abstract}

Key words: patterned ferromagnetic entities, magnetostrictive response, magneto-optics, atomic force microscopy, nanoscale imaging, magnetism dots,

\section{Introduction}

Patterned magnetic media are currently of great interest from the viewpoint of their potential application in novel devices for magnetic/ magneto-optic recording ${ }^{1), 2)}$. Features as small as $100 \mathrm{~nm}$ or less are produced currently by means of multi-step lithographic processes involving deposition and subsequent etching of a resist layer as the key technological operations. In our work, we use a simple effective and low-cost approach to such fabrication, namely, direct (resistless) patterning ${ }^{3), 4)}$ of small magnetic entities in alloyed layers of $\mathrm{Fe}-\mathrm{Cr}$ type via laser-induced phase changes. Various studies on nanocrystal-line Fe-based alloys ${ }^{5)-7)}$ emphasize the dominating role of superparamagnetic Fe-rich clusters in the behavior of the entire system, so that the alloys do not exhibit long-range magnetic order up to high $\mathrm{Fe}$ content $(70-75$ at. \%). However, in $\mathrm{Fe}_{70} \mathrm{Cr}_{30}$ alloys we found that local laser annealing gives rise to room-temperature ferromagnetic phase entities, in which the saturation magnetization is close to that in bulk $\mathrm{Fe}^{4), 8)}$. It is believed that on laser annealing the alloy components mix up in the liquid phase to yield, when cooled, a supersaturated solid solution with a high Curie temperature ${ }^{9}$.

In producing small magnetic features by laser processing the patterned features strongly depend on the parameters of the heating pulse. A high intensity or too long pulse duration causes blurring of the modified regions. To characterize unambiguously such magnetic features in an otherwise nonmagnetic matrix we require microscopic techniques such as the magnetic force microscopy (MFM). In addition to MFM, we here employ a newly developed technique for magnetic imaging, namely, detection of a local magnetostrictive response (MSR) by using a nonmagnetic probe of an atomic force microscope (AFM) in an applied a.c. field in situ ${ }^{10)}$. MSR measurements allow us to identify the locations in the matrix which have different magnetic properties. It is also important to point out that the spatial resolution of our MSR technique is comparable to that of a typical of AFM which is of the order of a few nanometers. The obtained MSR data are compared to the magnetization curves obtained by means of the longitudinal magneto-optic Kerr effect (MOKE). This comparison enables us to explain the observed MSR dependence on the magnetic moment orientation, the applied magnetic field direction and on the shape of the patterned entities.

\section{Experimental}

The Fe-Cr alloyed layers for subsequent processing were prepared on Si substrates by a $1.06 \mu \mathrm{m} \mathrm{Nd}$ :YAG laser ablation off separate $\mathrm{Fe}$, and $\mathrm{Cr}$ targets. Ferromagnetic resonance (FMR) studies show ${ }^{8)}$, that the as-prepared $\mathrm{Fe}_{70} \mathrm{Cr}_{30}$ mixtures did not possess any magnetic response at room temperatures, whereas a well-defined FMR signal appeared after irradiation by a one nanosecond laser pulse with fluence of about 0.1 $\mathrm{J} / \mathrm{cm}^{2}$. 
To produce small features on the above basis, the alloy mixture is irradiated by four interfering beams of a XeCl $(\lambda=308 \mathrm{~nm})$ narrowband $(0.04$ $\mathrm{cm}^{-1}$ ) excimer laser having a pulse duration of 10 $n s$. A coherent addition of four beams incident in two mutually orthogonal planes results in two-dimensional patterns of interference maxima and minima of the light intensity with a periodicity of $\lambda /(\sqrt{ } 2 \sin \theta)$, where $\theta$ is the angle of incidence. The patterned regions can have an anisotropic shape if (i) the beams are incident under different angles and (ii) the thermal diffusion length $(\sim 100-200 \mathrm{~nm})$ is small compared to the areas of the highest laser intensity in the interference maxima. The details of the interferometric laser annealing are given elsewhere ${ }^{11)}$.

In order to induce magnetostrictive surface oscillations of patterned samples, an ac magnetic field with the frequency in the range from 30 to $100 \mathrm{kHz}$, and with the amplitude $\mathrm{h}_{\sim}$, not more than a few Oe's is applied in situ. The frequency, $\omega$, was chosen so as to have $2 \omega$ close to the resonance frequency of the cantilever. The magnetostrictive deformations are quadratic in the direction cosines of the magnetization vector so that the deflection of the AFM tip at the second harmonics, $2 \omega$, can be associated with MSR under the probe scanning in the contact mode. The diagonal components of the deformation tensor are proportional to $\chi^{2} h_{\sim}{ }^{2} \cos 2 \omega t$, where $\chi$ is the a.c. susceptibility ${ }^{12)}$. Thus, a locally observable MSR may indicate the regions with different susceptibilities. The MSR studies were performed using a suitably modified "Burleigh" scanning probe microscope. In this apparatus we have added a facility to apply at the sample holder an alternating magnetic field in situ both in the sample plane and perpendicular to it. The amplitude of magnetostrictive oscillations (MSR) was measured in arbitrary units by using a lock-in amplifier.

\section{Results and Discussion}

Figure 1 shows (a) a topographic (AFM) image of patterned surface of a 15-nm thickness layer of $\mathrm{Fe}_{70} \mathrm{Cr}_{30}(\mathrm{Fe}-\mathrm{Cr})$ as well as (b) the corresponding MFM image collected after the sample was magnetized in the direction along the $X$ axis. These AFM/MFM images have been obtained using a Solver P47 apparatus (NT-MDT). The AFM image shows the crater-like regions located at the interference maxima. The crater shape is a result of the film melting in the interference maxima and the Fe-Cr liquid being expelled by a radial vapor pressure ${ }^{13)}$ from the center to the periphery. The viscosity of the $\mathrm{Fe}-\mathrm{Cr}$ liquid is (a)

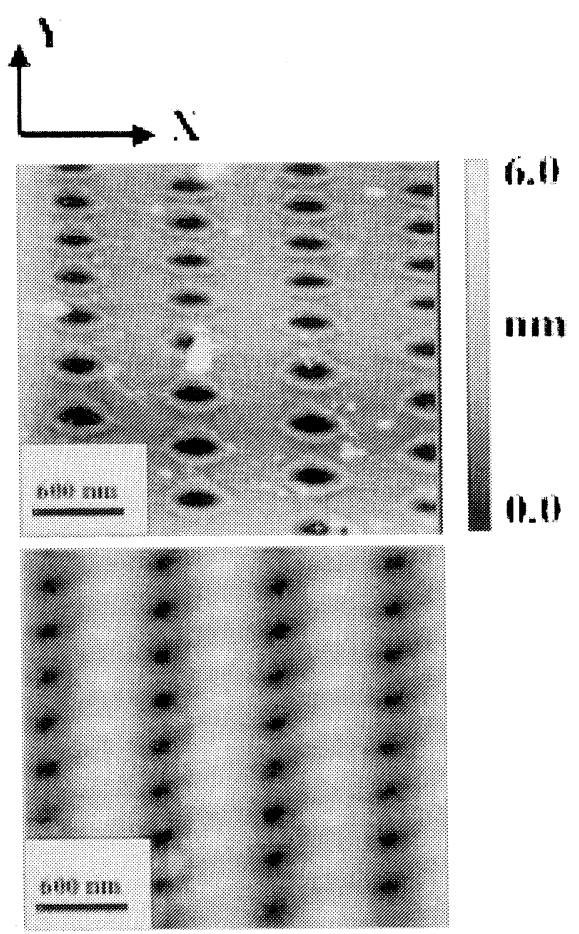

Fig.1 Topographic (a) and MFM (b) images of a 15-nm thickness $\mathrm{Fe}-\mathrm{Cr}$ layer patterned by means of interfering laser beams. The MFM image has been collected after magnetizing the sample in the direction along the $\mathrm{X}$ axis and indicates locally induced modifications in the magnetic properties.

high enough to prevent the formed surface profile from smoothing out over the cooling times which are of the order of $10^{-7} \mathrm{~s}$. The width of the crater walls (light contrast around the craters) is of $\sim 50$ $\mathrm{nm}$, and their height is of $\sim 5 \mathrm{~nm}$. The crater features depend on the laser fluences, so that the crater depth can reach the film thickness at fluences above $0.5 \mathrm{~J} / \mathrm{cm}^{2}$. The MFM image in Fig. 1(b) shows a periodic structure of dipoles: the dark and light contrast in the pattern indicate the poles of the patterned ferromagnetic regions. This observation provides the first and clear evidence of local laser-induced modifications in the magnetic properties of the alloys under study.

Figure 2 shows a topographic image (a) of the craters along with the two MSR images (b,c) obtained at frequency of $2 \omega(\omega=50.1 \mathrm{~Hz})$ for some value of the ac-field oriented along the long axis of the craters. The two MSR images were collected after prior mag magnetization of the sample along the long axis (b) as well as along their short axis (c) of the craters respectively by 


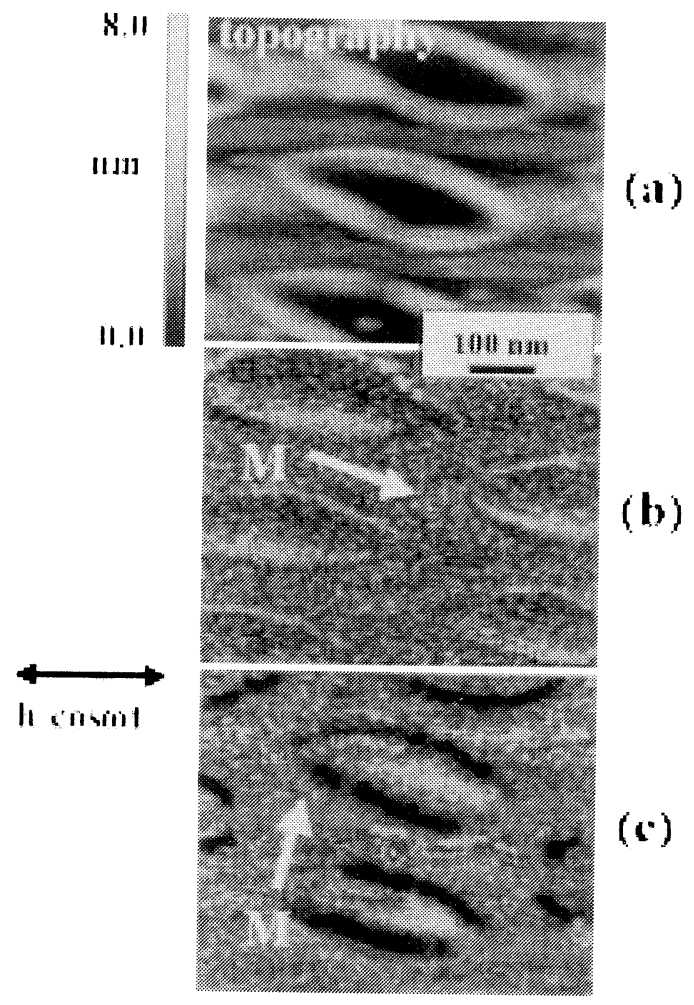

Fig.2 Topographic (a) and the corresponding MSR (b,c) images collected at the a.c. field with a frequency, $\omega$, of $50.1 \mathrm{kHz}$ after prior magnetization along the long axis (b) and the short axis (c) of craters. The orientation of the a.c. field and the magnetization in the craters, $M$, are indicated by arrows.

applying a static magnetic field of $1 \mathrm{kOe}$. First of all, note that the MSR was observed only within a very narrow frequency range $(\sim 10 \mathrm{~Hz})$ around the resonance frequency of the cantilever. It also remarkable that the topographic and magnetic features of the craters are in direct relationship. This similarity may have a simple explanation, considering that the crater walls is the most distinctive topographic feature, and that they also correspond to the maximum of the ferromagnetic matter which can have the largest MSR. It is interesting that the oscillation amplitude in the crater walls depends on the orientation of prior maglletization of the craters. When a sample is magnetized along the short axis of the craters, the wall oscillations are even smaller than those of the surrounding superparamagnetic medium [Fig.2(c)]. However, some enhancement of wall oscillations was observed after the sample was magnetized along the long axis of the craters. In the latter case, the oscillations became larger than those of the surrounding medium [Fig. 2 (b)]. It is useful to point out that MSR vanishes when the a.c. field is perpendicular to the sample plane. It should be noted here that the MSR was sensitive to the field reorientation in the film plane, as well.

Thus, the MSR technique presented here allows identification of locations with different magnetic properties. The magnetic properties of the crater walls differ from those of intra-crater regions and inter-crater medium. In an applied a.c. magnetic field, the oscillation amplitude of crater walls strongly depends on the field orientation and the prior direction of magnetization. Our conclusion hereof is that, in the patterned layers, the ferromagnetic phase is localized mainly within the crater rings in the topographic images.

However, it is unclear how to interpret the observed MSR behavior. To understand these results qualitatively, we have compared them to the MOKE data.

Figure 3 shows MOKE intensity as a function of a static magnetic field, $H$, for an as-prepared $\mathrm{Fe}-\mathrm{Cr}$ layer (a); the same layer that has the elongated craters $(b, c)$ such as in Figs.1 and 2. The MOKE intensity dependence on $\mathrm{H}$ for the

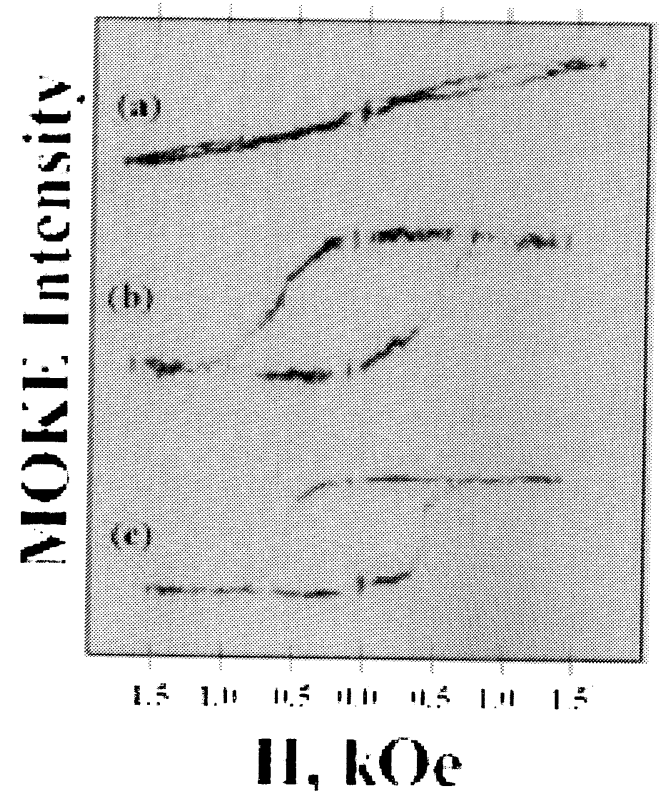

Fig.3 (a) Magnetization curve of an as-prepared $\mathrm{Fe}_{0.7} \mathrm{Cr}_{0.3}$ layer. (b,c) Hysteresis loops of a patterned sample, obtained at different orientation of the external field, $\mathrm{H}$, with respect to the crater axis: along the long axis (b) and along the short one (c). 
elongated craters was measured for two different orientations of $\mathrm{H}$, - along the long axis of craters (b) and along their short axis (c). We see that the magnetization curves of the patterned films possess the hysteresis features that are typical of ferromagnets, whereas the as-prepared film exhibits superparamagnetic-like (anhysteretic) behavior with a relative low static susceptibility, $(\mathrm{dM} / \mathrm{dH})_{\mathrm{H} \rightarrow 0}$, and some magnetization saturation at fields above $1 \mathrm{kOe}$. The hysteresis loops under study indicate a nearly full moment in the remanent state. This agrees well with the observed uniform magnetization of the craters in Fig.1 (b). Comparing the hysteresis loops measured along the long (b) and short (c) axes, one can see that the susceptibility is higher under magnetization along the long axis. Therefore, we see the enhancement of the oscillation amplitude at the ac-field applied along the long axis.

When the craters are magnetized along their short axis, the hysteresis loop has a nearly square shape, and the system is in its energy minimum so that the crater wall oscillations here are lowest. In greater detail, the peculiarities of the magnetic hysteresis of such patterned media were studied in Ref. 4. Here we only note that the observed hysteresis features can result from the influence of the superparamagnetic enviroment, which is believed to maximize when the external field is applied along the direction of close craters packing (axis $Y$ in Fig.1). In the context of this work, it is important to point out a qualitative relationship between the locally measured MSR by means of AFM and the data obtained by the conventional methods of magnetometry.

\section{Conclusion}

As a conclusion, we wish to emphasize that the local MSR detecting technique presented here is well suited for characterizing the composite (multiphase) media containing the regions with different susceptibilities. As applied to the systems under study, this technique is sensitive to the magnetism of the patterned features themselves as well as to that of the surrounding paramagnetic medium.

The observed MSR value dependence on the orientation of magnetic moments and on the shape of magnets can qualitatively be interpreted by comparison with the magnetization curves obtained by the longitudinal MOKE measurements.
Finally, we believe that the possibility for manipulating the MSR by external magnetic field can be utilized in the manufacturing process of small reversible magnetic memory cells. Our future work will focus on studying small objects that exhibit a large magnetostriction effect in combination with good soft magnetic properties.

\section{Acknowledgement}

This research was partially supported by the RFBR grant (\#01-02-16445). (NP) wishes to thank the materials science department at KTH for their hospitality during a visit. This work is partly funded by the Royal Swedish Academy of sciences.

\section{References}

1) S.Y. Chou, Proc. IEEE 85, 652 ( 1997)

2) R.M.H. New, R.F.W. Pease, and R.L. White, $J$. Vac. Sci. Technol. B 13, 1089 (1995).

3) M. Zheng, M. Yu, R. Skomski, S.H. Liou, D.J. Sellmyer, V.N. Petryakov, Yu.K. Verevkin, N.I. Polushkin, and N.N. Salashchenko, Appl. Phys. Lett. 79, 2606 (2001).

4) A.M. Alekseev, Yu.K. Verevkin, N.V. Vostokov, V.N. Petryakov, N.I. Polushkin, A.F. Popkov, and N.N. Salashchenko, JETP Letters, 73, 192 (2001).

5) C. Schwink, K. Emmerich, and U. Schulze. Z. Phys., B31, 385 (1978)

6) K. Takanashi, T. Sugawara, K. Hono, and H. Fujimori, J. Appl. Phys., 76, 6790 (1994).

7) D. Babonneau, J. Briatico, F. Petroff, T. Cabioc' h, and A. Naudon. J. Appl. Phys. 87, 3432 (2000).

$8)$ N.I. Polushkin, S.A. Gusev, M.N. Drozdov, Yu.K. Verevkin, and V.N. Petryakov, J. Appl. Phys., 81, 5478 (1997).

9) O. Kubaschewski. Iron-binary phase diagrams. Berlin: Springer, 1982 .

10) J. Wittborn, K.V. Rao, J. Nogues, and I.K. Schuller, Appl. Phys. Lett.,76, 2931(2000).

11) V.I. Bredikhin, Yu.K. Verevkin, E.Ya. Daume, S.P. Kuznetsov, O.A. Mal'shakova, V.N. Petryakov, N.V. Vostokov, and N.I. Polushkin, Quantum Electronics (Moscow) 30, 333(2000).

12) L.D. Landau, E.M. Lifshitz, and L.P. Pitaevskii, Electrodynamics of Continuous Media, $2^{\text {nd }}$ ed. (Butterworth-Heinenann, Oxford, England, 1984)

13) M. Allmen, Laser-beam interactions with materials. Berlin: Springer, 1987.

Received May 5, 2002; Accepted August 5, 2002 